\title{
Eosinophilic Esophagitis
}

National Cancer Institute

\section{Source}

National Cancer Institute. Eosinophilic Esophagitis. NCI Thesaurus. Code C27105.

Allergic inflammation of the esophagus. Morphologically, it is characterized by the presence of eosinophils infiltrating the esophageal epithelium. Patients present with swallowing difficulty and heartburn. 